\title{
Descriptive compact spaces and renorming
}

\author{
by \\ L. ONCINA and M. RAJA (Murcia)
}

\begin{abstract}
We study the class of descriptive compact spaces, the Banach spaces generated by descriptive compact subsets and their relation to renorming problems.
\end{abstract}

1. Introduction. Compact Hausdorff spaces which are fragmentable by a finer metric have been studied by many authors; see the book [7] for an account of this class of compacta. In this paper we shall consider a subclass $\mathfrak{D}$ of fragmentable compact spaces [26] that will allow us to construct an equivalent dual strictly convex norm on a dual Banach space $X^{*}$ if the dual unit ball lies in $\mathfrak{D}$. We have to introduce some terminology. Let $\left\{H_{i}: i \in I\right\}$ be a family of subsets of a topological space $(X, \tau)$. The family is said to be isolated if it is discrete in its union endowed with the relative topology, that is, if for every $i \in I$ and every $x \in H_{i}$, there is a $\tau$-neighbourhood $U$ of $x$ such that $H_{j} \cap U=\emptyset$ for every $j \in I, j \neq i$. If it is possible to pick $U$ from some given family $\mathcal{S} \subset \tau$, we say that the family is isolated with respect to $\mathcal{S}$. If there is a decomposition $I=\bigcup_{n=1}^{\infty} I_{n}$ such that every family $\left\{H_{i}: i \in I_{n}\right\}$ is isolated (with respect to $\mathcal{S}$ ), then the family $\left\{H_{i}: i \in I\right\}$ is said to be $\sigma$-isolated (with respect to $\mathcal{S}$ ). Finally, a family $\mathfrak{N}$ of subsets of $X$ is said to be a network for the topology of $X$ if every open set is a union of members of $\mathfrak{N}$.

Definition 1.1. A compact Hausdorff space $K$ is said to be a descriptive compact space if its topology has a $\sigma$-isolated network.

The class of topological spaces having a $\sigma$-isolated network generalizes in a natural way metrizable spaces (Bing-Nagata-Smirnov Theorem, see e.g. [16]). These spaces were first studied by Hansell in his pioneering work [10] recently published in [12]. He proved there, among other results, that if a topological space is fragmented by a finer metric, then it has a $\sigma$-isolated network if, and only if, it has a certain covering property, namely, the space is

2000 Mathematics Subject Classification: 46B26, 46B03, 46B20, 54D20.

Research partially supported by the D.G.E.S. grant PB98-0381. 
hereditarily weakly $\theta$-refinable. Descriptive Banach spaces have been studied in $[12,21]$ and also in the context of renorming theory in $[18,19,23-25]$.

The first clear example of a descriptive compact space one may come across is any metrizable compactum. Embeddings into $c_{0}(\Gamma)$ of Eberlein compacta show that they are descriptive, since $\left(c_{0}(\Gamma)\right.$, pointwise) has a $\sigma$-isolated network $[12,21]$. More generally, Gul'ko compact spaces are also known to be descriptive (see for instance [26]). Scattered compacta $K$ with $K^{\left(\omega_{1}\right)}=\emptyset$ are also descriptive: just consider as singletons the points of each relatively discrete set $\left\{K^{(\alpha)} \backslash K^{(\alpha+1)}: 0 \leq \alpha<\gamma\right\}$, where $\gamma<\omega_{1}$ is such that $K^{(\gamma)}=\emptyset$. Corson compact spaces defined by "almost disjoint families of sets" are also descriptive (see Remark 4.6), which includes an interesting compactum (Example 4.5), built by Argyros and Mercourakis [1]. On the other hand, the compactum $\left[0, \omega_{1}\right]$ is not descriptive $([12]$, see also Example 4.4).

Let us now turn our attention to renorming problems. Some results have recently been obtained showing that geometrical properties such as the existence of equivalent Kadec or locally uniformly rotund (LUR) norms in a Banach space $X$ can be characterized by the existence of certain types of networks of the norm topology which are $\sigma$-isolated for the weak topology of $X$ (LUR norms [18, 19, 24], dual LUR norms [24, 25] and Kadec norms [23]). Recently, in [8], it has been proved that the dual unit ball (with its weak* topology) is uniformly Eberlein if, and only if, the dual space has a $w^{*}$-UR equivalent norm, which is equivalent to $X$ having a uniformly Gateaux smooth equivalent norm. We shall introduce a general concept of $\tau$-LUR norm, for $\tau$ a locally convex topology.

Definition 1.2. A norm $\|\cdot\|$ on $X$ is said to be $\tau$-locally uniformly rotund at a point $x \in X$ if for every $\left(x_{n}\right) \subset X$ with $\lim _{n}\left\|x_{n}\right\|=\|x\|$ and $\lim _{n}\left\|x+x_{n}\right\|=2\|x\|$, we have $\tau-\lim _{n} x_{n}=x$.

A norm $\|\cdot\|$ on $X$ is said to be $\tau$-locally uniformly rotund ( $\tau$-LUR) if it is $\tau$-lower semicontinuous and $\tau$-locally uniformly rotund at every $x \in X$.

For the case of the weak topology, Moltó, Orihuela, Troyanski and Valdivia [19] proved that a $w$-LUR Banach space has an equivalent LUR norm. Mercourakis showed that the space $c_{1}\left(\Sigma^{\prime} \times \Gamma\right)$ has a pointwise-LUR equivalent norm. He used that fact to build an equivalent $w^{*}$-LUR norm in a dual of a WCD Banach space (see also [6]). The main result in [26] shows that a dual Banach space $X^{*}$ admits an equivalent $w^{*}$-LUR norm if, and only if, $\left(B_{X^{*}}, w^{*}\right)$ is a descriptive compact space.

In this paper we give sufficient and necessary conditions on a Banach space $X$ and a locally convex topology $\tau$ to obtain a $\tau$-LUR norm (equivalent or coarser) on $X$. For a total subspace $F \subset X^{*}$ we shall consider its associated norm $\mathfrak{p}_{F}(x)=\sup \left\{x^{*}(x): x^{*} \in B_{X^{*}} \cap F\right\}$. Recall that $F$ is 
called norming if $\mathfrak{p}_{F}$ is an equivalent norm on $X$. Our main result concerns "descriptively generated spaces":

Theorem 1.3. Let $X$ be a Banach space, $F \subset X^{*}$ a total subspace and $K \subset X$ a descriptive $\sigma(X, F)$-compact subset such that $X=\overline{\operatorname{span}}\|\cdot\|(K)$. Then:

(1) $X$ admits a coarser $\sigma(X, F)$-LUR norm and the topology $\sigma(X, F)$ on $X$ has a $\sigma$-isolated network.

(2) Moreover, if $K$ is fragmented by $\mathfrak{p}_{F}$, then $X$ admits a coarser norm which is $\mathfrak{p}_{F}-L U R$ and $\sigma(X, F)$-lower semicontinuous.

(3) The norms given in (1) and (2) can be taken equivalent to the original norm of $X$ if, and only if, $F$ is norming.

This theorem covers both the cases of weakly compactly generated Banach spaces and dual Banach spaces such that $\left(B_{X^{*}}, w^{*}\right)$ is a descriptive compactum. Since a weakly compact subset of a Banach space is descriptive and norm fragmented, we get Troyanski's result: a WCG Banach space is LUR renormable (see [6]). The theorem also applies to Banach spaces with a Markushevich basis. Indeed, if $\left\{x_{\alpha}, f_{\alpha}\right\}$ is an M-basis on $X$, and $F=\overline{\operatorname{span}}^{\|\cdot\|}\left\{f_{\alpha}\right\}$, then $\left\{x_{\alpha}\right\} \cup\{0\}$ is a descriptive $\sigma(X, F)$-compact set that generates $X$. Statement (2) above can also be deduced from the results of [25]. The topological properties of Banach spaces generated by a norm fragmentable compact space have recently been studied in [5].

Recall that descriptive compact spaces are fragmentable ([26], see Corollary 2.6 for a self-contained proof). In Section 2 we study the structure of a descriptive compactum with respect to a finer fragmenting metric. Section 3 is devoted to renorming, including the proof of Theorem 1.3. In the last section we show that the class of descriptive compacta has behaviour similar to the class of fragmentable compacta, studied by Ribarska [27] (see also [7]). Moreover, descriptive compact spaces have nicer properties than fragmentable ones (see Proposition 4.2). We also discuss some examples to show the scope of the class of descriptive compacta.

2. Spaces with $\sigma$-isolated network. Hansell's definition of descriptive topological spaces [12], later called isolated-analytic spaces in [11], is quite technical. In the case of a compact topological space, being descriptive in the sense of Hansell is equivalent to satisfying the condition of Definition 1.1.

The following definition has been used in [23-25].

Definition 2.1. Let $\mathcal{S}_{1}$ and $\mathcal{S}_{2}$ be families of subsets of a given set $X$. We say that $X$ has $P\left(\mathcal{S}_{1}, \mathcal{S}_{2}\right)$ with a sequence $\left(A_{n}\right)$ of subsets of $X$ if for 
every $x \in X$ and every $V \in \mathcal{S}_{1}$ with $x \in V$, there is $n \in \mathbb{N}$ and $U \in \mathcal{S}_{2}$ such that $x \in A_{n} \cap U \subset V$.

One can easily realize that this generalized property $P$ is also transitive, that is, if $X$ has $P\left(\mathcal{S}_{1}, \mathcal{S}_{2}\right)$ and $P\left(\mathcal{S}_{2}, \mathcal{S}_{3}\right)$ then $X$ has $P\left(\mathcal{S}_{1}, \mathcal{S}_{3}\right)$.

Very often one of families linked by the symbol $P($,$) is a topology$ on $X$. In this context, the topology generated by a given metric $d$ will also be denoted by $d$.

The following result links our property $P$ with the existence of $\sigma$-isolated networks. The implication (i) $\Leftrightarrow($ iii) appears in [10] for topologies.

TheOREm 2.2. Let $(X, \tau)$ be a regular topological space and let $\mathcal{S}$ be a subfamily of $\tau$. The following statements are equivalent:

(i) $\tau$ has a network which is $\sigma$-isolated with respect to $\mathcal{S}$.

(ii) There is a finer metric $d$ such that $X$ has $P(d, \mathcal{S})$.

(iii) There is a finer metric $d$ such that $X$ has $P(d, \mathcal{S})$ with a sequence of $\tau$-closed sets.

(iv) There exists a finer metric $d, \tau$-closed sets $A_{n}$ and families $\left\{U_{i}: i \in\right.$ $\left.I_{n}\right\}$ of $\tau$-open sets which are unions of sets from $\mathcal{S}$, such that the families $\left\{A_{n} \cap U_{i}: i \in I_{n}\right\}$ are disjoint and $\left\{A_{n} \cap U_{i}: n \in \mathbb{N}, i \in I_{n}\right\}$ is a network for $d$.

Proof. (i) $\Rightarrow$ (iii). The first step will be to show that there is a metric $d$ such that $X$ has $P(d, \tau)$ with a sequence of $\tau$-closed sets, so it is enough to assume that the network is simply $\sigma$-isolated. Let $\left\{H_{i}: i \in I\right\}$ be a network and $I=\bigcup_{n=1}^{\infty} I_{n}$, where each family $\left\{H_{i}: i \in I_{n}\right\}$ is isolated. Since $X$ is regular, the family $\left\{\bar{H}_{i}^{\tau}: i \in I\right\}$ is also a network for $\tau$. Take now $\tau$-open sets $U_{i}$ for $i \in I_{n}$ such that

$$
H_{i} \subset U_{i}, \quad U_{i} \cap \bigcup\left\{H_{j}: j \in I_{n}, j \neq i\right\}=\emptyset .
$$

Set $A_{n}^{1}={\overline{\bigcup_{i \in I_{n}} H_{i}}}^{\tau}$. Since $U_{i}$ is open, we have $H_{i} \subset A_{n}^{1} \cap U_{i} \subset \bar{H}_{i}^{\tau}$. This implies that $\left\{A_{n}^{1} \cap U_{i}: n \in \mathbb{N}, i \in I_{n}\right\}$ is a network for $\tau$.

Put $A_{n}^{2}=A_{n}^{1} \backslash \bigcup_{i \in I_{n}} U_{i}$. For every $n \in \mathbb{N}$, the family

$$
\mathfrak{B}_{n}=\left\{A_{n}^{2}, X \backslash A_{n}^{1}, A_{n}^{1} \cap U_{i}: i \in I_{n}\right\}
$$

is a partition of $X$. It is easy to see that $\bigcup_{n=1}^{\infty} \mathfrak{B}_{n}$ is a subbasis for a metrizable topology. Let $d$ be a compatible metric with that topology. It is clear that $d$ is finer than $\tau$ because $\bigcup_{n=1}^{\infty} \mathfrak{B}_{n}$ contains a network of $\tau$. On the other hand, every basic $d$-open set is a finite intersection of a $\tau$-open set with, possibly, sets of type $A_{n}^{1}$ and $A_{n}^{2}$. This shows that $X$ has $P(d, \tau)$ with the countable collection of the finite intersections of $A_{n}^{1}$ 's and $A_{n}^{2}$ 's.

Assume now that the network is $\sigma$-isolated with respect to $\mathcal{S}$. We claim that $X$ has $P(\tau, \mathcal{S})$ with a sequence of $\tau$-closed sets. As above consider $A_{n}^{1}={\overline{\bigcup_{i \in I_{n}}}}_{H_{i}}{ }^{2}$. Take $x \in X$ and some $\tau$-neighbourhood $U$ of $x$. Take a 
$\tau$-neighbourhood $V$ of $x$ such that $\bar{V}^{\tau} \subset U$. For some $n \in \mathbb{N}$, there is $i \in I_{n}$ such that $x \in H_{i} \subset V$. Take $S \in \mathcal{S}$ such that $x \in S$ and $H_{i} \cap S \subset V$. Then

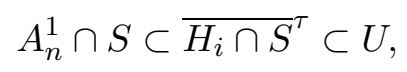

which proves the claim. Transitivity of $P$ shows that $X$ has $P(d, \mathcal{S})$ with a sequence of $\tau$-closed sets.

(iii) $\Rightarrow$ (iv). Assume that $X$ has $P(d, \mathcal{S})$ with a sequence $\left(A_{n}\right)$ of $\tau$-closed sets. Let $\left\{B_{j}: j \in J\right\}$ be a basis of the $d$-topology with $J=\bigcup_{n=1}^{\infty} J_{n}$, where every family $\left\{B_{j}: j \in J_{n}\right\}$ is discrete. This is possible by the Bing-NagataSmirnov Theorem [16]. Put

$$
I=J \times \mathbb{N}, \quad I_{n, m}=J_{n} \times\{m\}, \quad A_{n, m}=A_{m} .
$$

For $i=(j, m) \in I_{n, m}$ let $U_{i}$ the largest union of sets from $\mathcal{S}$ (maybe empty) such that $A_{m} \cap U_{i} \subset B_{j}$. Then $\left\{A_{n, m} \cap U_{i}: i \in I_{n, m}\right\}$ is disjoint and $\left\{A_{n, m} \cap U_{i}:(n, m) \in \mathbb{N} \times \mathbb{N}, i \in I_{n, m}\right\}$ is a network for $d$. Finally enumerate $\mathbb{N} \times \mathbb{N}$.

(iii) $\Rightarrow$ (ii) is trivial.

(ii) $\Rightarrow($ i). The same proof as in (iii) $\Rightarrow$ (iv) gives a network for $d$ which is $\sigma$-isolated with respect to $\mathcal{S}$. A network for $d$ is also a network for $\tau$ because the $d$-topology is finer that $\tau$.

Recall the definition of fragmentability, due to Jayne and Rogers [14].

Definition 2.3. Let $(X, \tau)$ be a topological space and $d$ a metric on $X$. We say that $X$ is fragmentable by $d$ if for every $\varepsilon>0$ and every nonempty $A \subset X$ there is $U \in \tau$ such that $A \cap U \neq \emptyset$ and $\operatorname{diam}(A \cap U)<\varepsilon$.

The following notion has been considered in topology, among the so called "covering properties" (see [3]).

Definition 2.4. A topological space $X$ is said to be weakly $\theta$-refinable (or weakly submetacompact) if every open cover of $X$ has a $\sigma$-isolated (not necessarily open) refinement. If every subspace of $X$ is weakly $\theta$-refinable, then $X$ is hereditarily weakly $\theta$-refinable.

The interest of the notion of hereditarily weakly $\theta$-refinable space is that it seems to be the most general and reasonable ingredient that allows one to pass from "scattered" properties to "isolated" ones. Compare this result with Theorem 2.2:

TheOREM 2.5. Let $X$ be a hereditarily Baire space and let $d$ be a finer metric on $X$. Then the following statements are equivalent:

(i) $X$ is hereditarily weakly $\theta$-refinable and fragmented by $d$.

(ii) $X$ has $P(d, \tau)$ with a sequence of $\tau$-closed sets.

Proof. (i) $\Rightarrow$ (ii) is shown in [26]. 
(ii) $\Rightarrow$ (i). By Theorem 2.2, $X$ has a $\sigma$-isolated network. It is easy to check that a topological space having a $\sigma$-isolated network is hereditarily weakly $\theta$-refinable. Let $X$ have $P(d, \tau)$ with a sequence $\left(A_{n}\right)$ of $\tau$-closed sets. Fix $\varepsilon>0$ and let $C \subset X$ be a nonempty $\tau$-closed set. Define

$$
C_{n}=\left\{x \in C \cap A_{n}: \exists U \in \tau, x \in U, \operatorname{diam}\left(A_{n} \cap U\right)<\varepsilon\right\} .
$$

Since $C=\bigcup_{n=1}^{\infty} C_{n}$, by the Baire property for some $n \in \mathbb{N}$ there exists $V \in \tau$ such that $\emptyset \neq C \cap V \subset \bar{C}_{n}^{\tau}$. In particular we can take $x \in C_{n} \cap V$. Let $U \in \tau$ be such that $x \in U$ and $\operatorname{diam}\left(A_{n} \cap U\right)<\varepsilon$. We have

$$
x \in C \cap V \cap U \subset \bar{C}_{n}^{\tau} \cap U \subset A_{n} \cap U
$$

and therefore $\operatorname{diam}(C \cap V \cap U)<\varepsilon$.

Corollary 2.6. Let $X$ be a hereditarily Baire space with a $\sigma$-isolated network. Then the finer metric d given by Theorem 2.2(iii) is a fragmenting metric.

Corollary 2.7. If $(X, \tau)$ is a regular hereditarily weakly $\theta$-refinable topological space fragmented by a finer metric $d$, then the $\tau$-Borel sets coincide with the $d$-Borel sets in $X$.

Proof. Property $P(d, \tau)$ with a sequence of $\tau$-Borel sets easily implies that every $d$-Borel set is a $\tau$-Borel set (see [23] for details).

3. Banach spaces and renorming. The first basic relation between $\tau$ LUR renormability and the existence of $\sigma$-isolated networks for some vector topology $\tau$ is given by the following result.

Proposition 3.1. Let $(X, \tau)$ be a locally convex space. If $X$ admits a $\tau$-LUR norm, then $(X, \tau)$ has a network which is $\sigma$-isolated with respect to $\tau$-open halfspaces.

Proof. In [19] it is proved that the weak topology of a $w$-LUR Banach space has a $\sigma$-isolated network. For the weak* topology of a $w^{*}$-LUR Banach space this is shown in [26] using a different approach. Both methods can be easily adapted to prove that if $X$ has a $\tau$-LUR norm, then $(X, \tau)$ has a $\sigma$-isolated network.

By Theorem 2.2 we may consider a finer metric $d$ defined on $X$ such that $X$ has $P(d, \tau)$. In order to prove the proposition, we need to show that $X$ has $P(d, \mathcal{S})$, where $\mathcal{S}$ denotes the family of $\tau$-open halfspaces. The transitivity of $P$ implies that it is enough to prove that $X$ has $P(\tau, \mathcal{S})$.

Fix $x \in X$ and a $\tau$-neighbourhood $U$ of $x$. We claim that there exist two rational numbers $0<s<r$ with $s<\|x\|<r$ such that the inequalities

$$
s<\|y\|<r \quad \text { and } \quad 2 s<\|x+y\|<2 r
$$


imply that $y \in U$. If not, then we could obtain a sequence $y_{n} \in X \backslash U$ such that

$$
\lim _{n}\left\|y_{n}\right\|=\|x\|, \quad \lim _{n}\left\|x+y_{n}\right\|=2\|x\|,
$$

which would contradict the fact that $\|\cdot\|$ is $\tau$-LUR. By the Hahn-Banach Theorem, we can find a $\tau$-open halfspace $H$ such that $x \in H$ and $B[0, s] \cap$ $H=\emptyset$. Now, if $y \in B[0, r] \cap H$, then $s<\|(x+y) / 2\|<r$, so $y \in U$. This proves that $X$ has $P(\tau, \mathcal{S})$.

The following result is in [26].

THEOREM 3.2. Let $K$ be a descriptive compact space and let $d$ be a finer metric fragmenting $K$. Then there is an equivalent dual norm $\|\cdot\|$ on $C(K)^{*}$ such that for every bounded d-continuous function $f: K \rightarrow X$ with values in a normed space,

$$
\lim _{n}\left\|\int f d \mu_{n}-\int f d \mu\right\|=0
$$

whenever the measures $\mu, \mu_{n} \in C(K)^{*}$ are such that $\lim _{n}\left\|\mu_{n}\right\|=\|\mu\|$ and $\lim _{n}\left\|\mu+\mu_{n}\right\|=2\|\mu\|$. In particular, $\|\cdot\|$ is a $w^{*}-L U R$ norm.

It follows from the previous result that a compact Hausdorff space is descriptive if, and only if, it embeds as a weak ${ }^{*}$-compact subset of a $w^{*}$-LUR dual Banach space.

The following is a version for $w^{*}$-LUR norms of the transfer technique of Godefroy, Troyanski, Whitfield and Zizler [6, Theorem II.2.1] developed for LUR norms.

Proposition 3.3. Let $T: Y^{*} \rightarrow X^{*}$ be a $w^{*}-w^{*}$-continuous linear operator between dual Banach spaces. If the norm of $Y^{*}$ is $w^{*}-L U R$, then $X^{*}$ has an equivalent dual norm which is $w^{*}-L U R$ at the points of $\overline{T\left(Y^{*}\right)}\|\cdot\|$.

Proof. If $T$ were surjective, it is not difficult to prove that an equivalent $w^{*}$-LUR norm $\|\cdot\|$ on $X^{*}$ would be defined by the formula

$$
\left\|x^{*}\right\|=\inf \left\{\left\|y^{*}\right\|: T\left(y^{*}\right)=x^{*}\right\} .
$$

In the general case, the construction can be done as follows. For every $k \in \mathbb{N}$ define an equivalent dual norm $\|\cdot\|_{k}$ on $X^{*}$ by the formula

$$
\left\|x^{*}\right\|_{k}^{2}=\inf \left\{\left\|x^{*}-T\left(y^{*}\right)\right\|^{2}+k^{-1}\left\|y^{*}\right\|^{2}: y^{*} \in Y^{*}\right\} .
$$

Notice that due to the weak* continuity of the map and the lower semicontinuity of the norms, the infimum is attained. Define

$$
\left\|x^{*}\right\|^{2}=\sum_{k=1}^{\infty} 2^{-k}\left\|x^{*}\right\|_{k}^{2} .
$$

It is not difficult to show that $\|\cdot\|$ is an equivalent norm. In order to show that it is $w^{*}$-LUR we shall follow the proof of [6, Theorem II.2.1]. So assume 
$x^{*} \in \overline{T\left(Y^{*}\right)}\|\cdot\|$ and $\left(x_{n}^{*}\right) \subset X^{*}$ are such that

$$
\lim _{n}\left(2\left\|x^{*}\right\|+2\left\|x_{n}^{*}\right\|-\left\|x^{*}+x_{n}^{*}\right\|\right)=0 .
$$

Given $\varepsilon>0$ and $x \in B_{X}$, let $y^{*} \in Y^{*}$ be such that for $k$ large enough,

$$
\left\|x^{*}\right\|_{k}^{2}=\left\|x^{*}-T\left(y^{*}\right)\right\|^{2}+k^{-1}\left\|y^{*}\right\|^{2} \leq \varepsilon / 4,
$$

in particular $\left\|x^{*}-T\left(y^{*}\right)\right\| \leq \varepsilon / 4$. For this fixed $k$, let $y_{n}^{*} \in Y^{*}$ be such that

$$
\left\|x_{n}^{*}\right\|_{k}^{2}=\left\|x_{n}^{*}-T\left(y_{n}^{*}\right)\right\|^{2}+k^{-1}\left\|y_{n}^{*}\right\|^{2} .
$$

Following a standard convexity argument we obtain

$$
\begin{gathered}
\lim _{n}\left\|x_{n}^{*}-T\left(y_{n}^{*}\right)\right\|=\left\|x^{*}-T\left(y^{*}\right)\right\|, \\
\lim _{n}\left(2\left\|y^{*}\right\|^{2}+2\left\|y_{n}^{*}\right\|-\left\|y^{*}+y_{n}^{*}\right\|\right)=0 .
\end{gathered}
$$

Now since $\|\cdot\|$ on $Y^{*}$ is $w^{*}$-LUR, (2) implies $y_{n}^{*} \rightarrow y^{*}$ in the weak* topology. Also, for $n \geq n_{0},\left|\left(T\left(y_{n}^{*}-y^{*}\right)\right)(x)\right| \leq \varepsilon / 4$, and by $(1),\left|\left(T\left(y_{n}^{*}\right)-x_{n}^{*}\right)(x)\right| \leq \varepsilon / 2$. So $\left|\left(x^{*}-x_{n}^{*}\right)(x)\right| \leq \varepsilon$ as we wanted.

Corollary 3.4. Continuous images of descriptive compacta are descriptive.

Proof. Let $T: K_{1} \rightarrow K_{2}$ be a continuous surjection and suppose that $K_{1}$ is descriptive. The map $T$ can be extended to a linear $w^{*}-w^{*}$-continuous surjective operator $\widetilde{T}: C\left(K_{1}\right)^{*} \rightarrow C\left(K_{2}\right)^{*}$. Since $C\left(K_{1}\right)^{*}$ has an equivalent $w^{*}$ LUR norm, by the former proposition $C\left(K_{2}\right)^{*}$ is also $w^{*}$-LUR renormable, and this implies that $K_{2}$ is descriptive.

REMARK 3.5. More generally, it is known that the properties of having a $\sigma$-isolated network and being hereditarily weakly $\theta$-refinable are preserved under perfect maps [4].

Corollary 3.6. Let $X$ and $Y$ be Banach spaces and let $F \subset X^{*}$ be a total subspace. Assume that $Y^{*}$ is $w^{*}-L U R$ and there is a bounded linear operator $T: Y^{*} \rightarrow X$ with dense range which is also $w^{*}-\sigma(X, F)$-continuous. Then there exists a coarser norm on $X$ which is $\sigma(X, F)-L U R$ (the norm can be taken equivalent if and only if $F$ is norming).

Proof. We may consider $(X, \sigma(X, F))$ as a topological subspace of $\left(F^{*}, w^{*}\right)$. We shall regard $T$ as an operator into $F^{*}$. Clearly $T$ is bounded and $w^{*}-w^{*}$-continuous. Let $\|\cdot\|^{*}$ be the dual norm on $F^{*}$. Since the restriction to $X$ of $\|\cdot\|^{*}$ is $\|\cdot\|_{F}$ and this norm is coarser than the norm of $X$ we get $X \subset \overline{\operatorname{span}}\left\|^{\|}\right\|^{*}\left(T Y^{*}\right)$. The application of Proposition 3.3 will give a dual norm on $F^{*}$ (so equivalent to $\|\cdot\|^{*}$ ) which is $w^{*}$-LUR at the points of $X$ and the restriction to $X$ of this norm is the desired $\sigma(X, F)$-LUR norm.

Denote by $\overline{\operatorname{aco}}^{w^{*}}(K)$ the $w^{*}$-closed absolutely convex hull of a subset $K \subset X^{*}$. The following is an easy consequence of Corollary 3.4. 
Corollary 3.7. Let $K$ be a $w^{*}$-compact subset of a dual Banach space $X^{*}$. If $K$ is descriptive, then $\overline{\mathrm{aco}^{*}}(K)$ is also descriptive.

Proof. By [26], the unit ball of $C(K)^{*}$ is a descriptive compactum. Each point of $\overline{\operatorname{aco}} w^{*}(K)$ is the barycenter of some measure from $B_{C(K)^{*}}$ and the map is continuous, thus $\overline{\mathrm{aco}} w^{*}(K)$ is the continuous image of a descriptive compactum.

We are now able to prove our Main Theorem from the introduction.

Proof of Theorem 1.3. As in the proof of Corollary 3.6 we may embed $X$ into the dual space $F^{*}$. Thus, without loss of generality we shall assume that $K \subset X^{*}$ is a descriptive $w^{*}$-compact subset. We have to prove that $X^{*}$ admits an equivalent dual norm which is $w^{*}$-LUR at the points of $\overline{\operatorname{span}}^{\|\cdot\|}(K)$. Consider the operator $T: C(K)^{*} \rightarrow F^{*}$ defined by $T(\mu)=\int \mathbb{I} d \mu$.

Statement (1) follows by direct application of Proposition 3.3.

If $K$ is fragmented by $\mathfrak{p}_{F}$, that is, the norm of $F^{*}$, then we shall use Theorem 3.2 in its full generality. In that case, the $w^{*}$-LUR norm $\|\cdot\|$ on $C(K)^{*}$ given by the theorem has the following property: if

$$
\lim _{n}\left\|\mu_{n}\right\|=\|\mu\|, \quad \lim _{n}\left\|\mu_{n}+\mu\right\|=2\|\mu\| \quad \text { for } \mu, \mu_{n} \in C(K)^{*},
$$

then

$$
\lim _{n}\left\|T\left(\mu_{n}-\mu\right)\right\|=0 .
$$

Minor changes in the proof of Proposition 3.3 show that the norm $\|\cdot\|$ on $F^{*}$ is LUR.

To prove (3) observe that the norms obtained in $X$ are equivalent to $\mathfrak{p}_{F}$, and that norm is equivalent to the norm of $X$ if, and only if, $F$ is norming. On the other hand, if $X$ has an equivalent $\sigma(X, F)$-LUR norm, then $F$ should be a norming subspace.

Corollary 3.8. Let $X$ be a Banach space, $F \subset X^{*}$ a total subspace and $K \subset X$ a descriptive $\sigma(X, F)$-compact subset such that $X=\overline{\operatorname{span}}\|\cdot\|(K)$. Then $X$ admits an equivalent rotund norm.

Proof. Let $\|\cdot\|_{1}$ be the coarser $\sigma(X, F)$-LUR norm given by Theorem 1.3. It is easy to verify that the norm $\|\cdot\|_{2}=\|\cdot\|+\|\cdot\|_{1}$ is an equivalent rotund norm on $X$.

The proof of Theorem 1.3 actually gives the following.

Corollary 3.9. If $X^{*}$ is a dual Banach space and $K \subset X^{*}$ is a descriptive $w^{*}$-compact subset, then $X^{*}$ has an equivalent dual norm which is $w^{*}-L U R$ at the points of $\overline{\operatorname{span}}\|\cdot\|(K)$. Moreover, if $\overline{\operatorname{span}}\|\cdot\|(K)=X^{*}$ then $\left(B_{X^{*}}, w^{*}\right)$ is also a descriptive compact space.

We also collect the stability properties of the class of Banach spaces with descriptive dual ball. 
Proposition 3.10. The class of Banach spaces with descriptive dual unit ball, which we shall denote by $\mathfrak{D}_{*}$, has the following properties:

(i) If $X \in \mathfrak{D}_{*}$ and $T: X \rightarrow Y$ is a bounded linear operator such that $T(X)$ is dense in $Y$, then $Y \in \mathfrak{D}_{*}$. In particular $\mathfrak{D}_{*}$ is stable under quotients.

(ii) If $Y \in \mathfrak{D}_{*}$ and $T: X \rightarrow Y$ is a bounded linear operator such that $T^{*}\left(Y^{*}\right)$ is dense in $X^{*}$, then $X \in \mathfrak{D}_{*}$. In particular $\mathfrak{D}_{*}$ is stable under closed subspaces.

(iii) If $X_{i} \in \mathfrak{D}_{*}$ for $i \in I$ then $\bigoplus_{i \in I} X_{i} \in \mathfrak{D}_{*}$ for $c_{0}$ and $\ell_{p}$ sums where $1<p<\infty$. If $I$ is countable, the result also holds for $p=1$.

Proof. (i) $T^{*}$ is one-to-one and therefore $T^{*}\left(\left(B_{Y^{*}}, w^{*}\right)\right)$ is homeomorphic to a $w^{*}$-compact subset of $X^{*}$, hence descriptive.

(ii) follows from Proposition 3.3.

(iii) Fix, on each space $X_{i}$, a norm such that the dual norm on $X_{i}^{*}$ is $w^{*}$-LUR. For the $c_{0}$ sum we may define an equivalent norm $\|\cdot\|$ on

$$
\left(\bigoplus_{i \in I}^{c_{0}} X_{i}\right)^{*}=\bigoplus_{i \in I}^{\ell_{1}} X_{i}^{*}
$$

by the formula

$$
\left\|\left(x_{i}^{*}\right)_{i \in I}\right\|^{2}=\left(\sum_{i \in I}\left\|x_{i}^{*}\right\|\right)^{2}+\sum_{i \in I}\left\|x_{i}^{*}\right\|^{2} .
$$

It is not difficult to check that the norm $\|\cdot\|$ is an equivalent $w^{*}$-LUR norm. For the $\ell_{p}$ sum with $p \in(1, \infty)$, the $\ell_{q}$ sum of the dual spaces, where $q$ is the conjugate exponent, is $w^{*}$-LUR. For $I$ countable, it is easy to verify that the unit ball of $\left(\bigoplus_{i \in I}^{\ell_{1}} X_{i}\right)^{*}=\bigoplus_{i \in I}^{\ell_{\infty}} X_{i}^{*}$ is homeomorphic to the descriptive compact space $\prod_{i \in I} B_{X_{i}^{*}}$ (see Proposition 4.1).

REMARK 3.11. The class $\mathfrak{D}_{*}$ fails to have the three space property. In $[7$, Theorem 2.3.1] there is given an example of a Banach space $X$ which is not weak Asplund and has a separable subspace $Y$ such that $X / Y$ is Asplund and WCG.

4. Topological properties of descriptive compact spaces. In this section we study how "nice" the topology of a descriptive compactum is, and the behaviour under topological operations of the class of descriptive compacta. We shall also discuss some examples.

The following proposition puts together some stability properties of the class of descriptive compact spaces. Notice that fragmentable compacta have the same properties [7].

Proposition 4.1. Denote by $\mathfrak{D}$ the class of descriptive compact spaces. The class $\mathfrak{D}$ is stable under closed subspaces, countable products, one-point 
compactifications of discrete collections, and continuous images. If $K \in \mathfrak{D}$, then $B_{C(K)^{*}} \in \mathfrak{D}$. Finally, if $K$ is compact, $d$ a lower semicontinuous metric on $K$ and there are closed subsets $K_{n} \subset K$ such that $K_{n} \in \mathfrak{D}$ and $K=$ ${\overline{\bigcup_{n=1}^{\infty} K_{n}}}^{d}$, then $K \in \mathfrak{D}$.

Proof. The nontrivial properties follow from Corollary 3.4 and Theorem 3.2. Only the last one needs a proof. Assume that $d$ is a lower semicontinuous metric on $K$. By a result in [13] we may assume that $K$ is a $w^{*}$-compact subset of a dual $X^{*}$ and $d$ is induced by the norm metric. Take

$$
K_{0}=\bigcup_{n=1}^{\infty} n^{-1} K_{n} \cup\{0\},
$$

which is a descriptive $w^{*}$-compact subset of $X^{*}$. Corollary 3.9 implies that there is a dual norm on $X^{*}$ which is $w^{*}$-LUR at the points of $Y=\overline{\operatorname{span}}\|\cdot\|\left(K_{0}\right)$. Proposition 3.1 shows that $(Y, \sigma(Y, X))$ has a $\sigma$-isolated network. The hypothesis implies that $K$ is a subset of $Y$, therefore it is a descriptive compactum.

A topological space $X$ is said to be Fréchet-Urysohn if every cluster point of some subset $A \subset X$ is the limit of a sequence in $A$. The following result shows that descriptive compacta are close to Fréchet-Urysohn spaces.

Proposition 4.2. If $K$ is a descriptive compact space, then:

(i) $K$ is sequentially compact.

(ii) Countably compact subsets of $K$ are closed.

(iii) Hereditarily separable closed subsets of $K$ are metrizable.

Proof. (i) Any fragmentable compact space is sequentially compact $[7,20]$.

(ii) In $[3$, Theorem 9.2] it is proved that any weakly $\theta$-refinable countably compact space is compact.

(iii) follows from a much more general result [15, Corollary 9].

Corollary 4.3. A descriptive compact space has countable tightness and its sequentially closed subsets are closed.

A straightforward consequence of this corollary is the following example of a nondescriptive compactum (see also [12]).

Example 4.4. The interval $\left[0, \omega_{1}\right]$ is not descriptive.

Alternative ways to obtain the same conclusion is to prove that the Borel sets in $\left[0, \omega_{1}\right]$ do not coincide with the Borel sets for the discrete topology [29] and to apply Corollary 2.7; or to prove that $\left[0, \omega_{1}\right]$ is not a Gruenhage space [28]. The "long James space" $J\left(\omega_{1}\right)$ (see [2]) is a bidual Banach space with the Radon-Nikodym property which contains a weak* compact subset homeomorphic to $\left[0, \omega_{1}\right]$, so its unit ball cannot be descriptive. 
The unit ball of a dual space having a strictly convex dual norm is weak* fragmentable. Neither fragmentable nor Radon-Nikodym compacta [20] can be characterized by embeddings into dual Banach spaces with a strictly convex dual norm. Indeed, small changes in the proof of $[6$, Theorem VII.5.2] imply that the compact space $\left[0, \omega_{1}\right]$ does not embed into a dual Banach space with an equivalent strictly convex dual norm. We do not know if $C(K)^{*}$ can be renormed with a strictly convex dual norm when $K$ is a fragmentable compact space not containing a copy of $\left[0, \omega_{1}\right]$.

There are separable, nonmetrizable and scattered compacta $K$ such that $K^{(3)}=\emptyset$ (see e.g. [6, Example VI.8.7]). In consequence, there exist descriptive compact spaces which are not Gul'ko, nor even Corson. Recall that a compact space is called Corson if it is homeomorphic to a subset of $[0,1]^{\Gamma}$ made up of elements with countable support, and it is called Rosenthal if it is homeomorphic to a subset of functions of first Baire class on a Polish space. Since Gul'ko compacta are Corson (see e.g. [6, 7]), the following example is interesting to distinguish between those classes.

EXAmPle 4.5. There exists a compact Hausdorff space $K$ which is Corson, Rosenthal, not Gul'ko and descriptive.

Proof. That compactum was built by Argyros and Mercourakis [1] and we only have to prove its descriptiveness. To do that we use the construction of the compactum as presented in [7, Section 7.3]. Consider the sets $D_{n}$ of continuous functions on $K$ defined for $n \geq 2$, and take $D=\bigcup_{n \geq 2} D_{n}$ (the set $D$ in [7] also contains the constant function 1, but for our argument it is better to avoid it). A bounded linear operator $T: C(K)^{*} \rightarrow c_{0}(D)$ is defined. In the following, consider $C(K)^{*}$ with the weak* and $c_{0}(D)$ with the pointwise topologies, which also make $T$ continuous. In particular, $T(K)$ is Eberlein. From the definitions of $K$ and $T$ it follows easily that $L=$ $\{x \in K: T(x)=0\}$ is the one-point compactification of a discrete set and $T$ is a homeomorphism from $K \backslash L$ onto $T(K) \backslash\{0\}$. We deduce that the relative topologies on the sets $L$ and $K \backslash L$ have a $\sigma$-isolated network, and therefore $K$ is descriptive.

REMARK 4.6. Using a similar argument, it is not difficult to show that any Corson compact space defined by an almost disjoint family of subsets of $\mathbb{N}$ (for the definition see $[1,17]$ ) is descriptive.

We finish with an approximation to [6, Problem VII.2], where topological conditions on the bidual ball $B_{X^{* *}}$ are suggested to guarantee that $X$ has an equivalent LUR norm.

Proposition 4.7. Let $X$ be a Banach space such that $\left(B_{X^{* *}}, w^{*}\right)$ is descriptive. Then $\left(B_{X^{* *}}, w^{*}\right)$ is a compact Fréchet-Urysohn space and $X$ has an equivalent $L U R$ norm. 
Proof. The restriction of a $w^{*}$-LUR norm on $X^{* *}$ to $X$ is a $w$-LUR norm, thus $X$ has an equivalent LUR norm by [19]. Since $B_{X^{* *}}$ is sequentially compact, $X$ cannot contain an isomorphic copy of $l_{1}(\mathbb{N})$. If $A \subset B_{X^{* *}}$ and $x^{*} \in \bar{A}^{w^{*}}$, then since $B_{X^{* *}}$ has countable tightness, there is $A_{0} \subset A$ countable such that $x^{*} \in \bar{A}_{0}^{w^{*}}$. Using again the countable tightness, take a separable subspace $X_{0} \subset X$ such that $A_{0} \subset \bar{X}_{0}^{w^{*}}$. This implies that we can work inside $X_{0}^{* *}$. By a well known result of Rosenthal, $B_{X_{0}^{* *}}$ is FréchetUrysohn, thus we can find a sequence in $A_{0}$ weak* convergent to $x^{*}$.

The following example shows that not much more can be expected. The James Tree Space $J T$ (see e.g. [9]) is a separable space such that $J T^{*}$ is not separable and $J T^{* *}$ is isomorphic to $J T \oplus l_{2}(\Gamma)$ with $\Gamma$ uncountable, so $J T^{* *}$ is WCG and thus $B_{J T^{* *}}$ endowed with the weak* topology is a descriptive, Radon-Nikodym, Rosenthal, separable and nonmetrizable compact space.

Now [6, Problem VII.2] can be rewritten as follows: If a compact space is fragmentable and Corson, will it be descriptive? If the fragmenting metric is lower semicontinuous the answer is yes as a consequence of a result by Orihuela, Schachermayer and Valdivia [22], because in this case the compactum is Eberlein.

\section{References}

[1] S. Argyros and S. Mercourakis, On weakly Lindelöf Banach spaces, Rocky Mountain J. Math. 23 (1993), 395-446.

[2] R. D. Bourgin, Geometric Aspects of Convex Sets with the Radon-Nikodym Property, Lecture Notes in Math. 993, Springer, 1983.

[3] D. K. Burke, Covering properties, in: Handbook of Set-Theoretic Topology, Elsevier, 1984, 347-422.

[4] D. K. Burke and R. W. Hansell, Perfect maps and relatively discrete collections, in: Papers on General Topology and Applications (Amsterdam, 1994), New York Acad. Sci., New York, 1996, 54-56.

[5] B. Cascales, I. Namioka, and J. Orihuela, The Lindelöf property in Banach spaces, Studia Math. 154 (2003), 165-192.

[6] R. Deville, G. Godefroy, and V. Zizler, Smoothness and Renormings in Banach Spaces, Pitman Monogr. Surveys Pure Appl. Math., Longman, 1993.

[7] M. Fabian, Weak Asplund Spaces, Wiley, 1997.

[8] M. Fabian, G. Godefroy, and V. Zizler, The structure of uniformly Gateaux smooth Banach spaces, Israel J. Math. 124 (2001), 243-252.

[9] P. Habala, P. Hájec, and V. Zizler, Introduction to Banach Spaces, Charles Univ. Press, Prague, 1998.

[10] R. W. Hansell, Descriptive sets and the topology of nonseparable Banach spaces, preprint, 1989.

[11] —, Descriptive topology, in: Recent Progress in General Topology (Prague, 1991), North-Holland, Amsterdam, 1992, 275-315. 
[12] R. W. Hansell, Descriptive sets and the topology of nonseparable Banach spaces, Serdica Math. J. 27 (2001), 1-66.

[13] J. E. Jayne, I. Namioka, and C. A. Rogers, Norm fragmented weak ${ }^{*}$ compact sets, Collect. Math. 41 (1990), 133-163.

[14] J. E. Jayne and C. A. Rogers, Borel selectors for upper semicontinuous set-valued maps, Acta Math. 155 (1985), 41-79.

[15] I. Kortezov, Fragmentability and cardinal invariants, Topology Appl. 101 (2000), 93-106.

[16] K. Kuratowski, Topology, Vol. I, PWN-Polish Sci. Publ., Warszawa, 1966.

[17] S. Mercourakis and S. Negrepontis, Banach spaces and topology II, in: Recent Progress in General Topology, M. Hušek and J. van Mill (eds.), Elsevier, 1992, 493-536.

[18] A. Moltó, J. Orihuela, and S. Troyanski, Locally uniformly rotund renorming and fragmentability, Proc. London Math. Soc. 76 (1997), 614-640.

[19] A. Moltó, J. Orihuela, S. Troyanski, and M. Valdivia, On weakly locally uniformly rotund Banach spaces, J. Funct. Anal. 163 (1999), 252-271.

[20] I. Namioka, Radon-Nikodym compact spaces and fragmentability, Mathematika 34 (1989), 258-281.

[21] L. Oncina, The JNR property and the Borel structure of a Banach space, Serdica Math. J. 26 (2000), 13-32.

[22] J. Orihuela, W. Schachermayer, and M. Valdivia, Every Radon-Nikodym Corson compact space is Eberlein compact, Studia Math. 98 (1991), 157-174.

[23] M. Raja, Kadec norms and Borel sets in a Banach space, ibid. 136 (1999), 1-16.

[24] —, Locally uniformly rotund norms, Mathematika 46 (1999), 343-358.

[25] —, On dual locally uniformly rotund norms, Israel J. Math. 129 (2002), 77-91.

[26] - Weak* locally uniformly rotund norms and descriptive compact spaces, J. Funct. Anal. 197 (2003), 1-13.

[27] N. K. Ribarska, Internal characterization of fragmentable spaces, Mathematika 34 (1987), 243-257.

[28] -, A Radon-Nikodym compact which is not a Gruenhage space, C. R. Acad. Bulgare Sci. 41 (1988), 9-11.

[29] M. Talagrand, Pettis integral and measure theory, Mem. Amer. Math. Soc. 307 (1984).

Departamento de Matemáticas

Universidad de Murcia

Campus de Espinardo

30100 Espinardo, Murcia, Spain

E-mail: luis@um.es

matias@um.es

Received September 3, 2002

Revised version February 6, 2004 